ORIGINAL ARTICLE

\title{
The Abbreviated Psychopathy Measure-2: associations with normal-range and maladaptive personality traits
}

\author{
Robert A. Semel \\ Independent researcher
}

\section{BACKGROUND}

The aim of the study was to validate an updated form of the Abbreviated Psychopathy Measure which is based on the triarchic model of psychopathy. Revisions were made to improve indexing of the triarchic scales. The study focused on examining the relationships between the APM-2 scales with lower-order personality traits associated with psychopathy, as well as with antisocial intent, a correlate of antisocial behavior.

\section{PARTICIPANTS AND PROCEDURE}

A convenience sample of participants $(N=190)$ was recruited from Amazon's Mechanical Turk (MTurk). Construct validity of the APM-2 scales was examined using Pearson's $r$ correlation and multiple regression analyses to determine the relationships between APM-2 scales and criterion measures.

\section{RESULTS}

The APM-2 Total score was associated at moderate to high levels with core personality features associated with psychopathy. APM-2 Boldness was associated with both positive adjustment (social potency, emotional stability), and negative adjustment (both dangerous and calculated sensation seeking). APM-2 Meanness was associated with measures of callousness, hostile aggression, and manipulativeness, as well as with a measure of antisocial intent. APM-2 Disinhibition was associated with measures of impulsive, norm violating behavior, negative emotional disposition, and antisocial intent.

\section{CONCLUSIONS}

The associations between APM-2 scales and personality traits replicated certain key findings reported in the literature regarding psychopathy-relevant traits indexed by the Triarchic Psychopathy Measure (TriPM). Thus, this study provides a preliminary indication, albeit with a limited range of personality and antisocial behavior variables, that the nomological networks of the APM-2 scales may parallel the nomological networks of the TriPM scales.

\section{KEY WORDS}

psychopathic personality; triarchic model; five-factor model of personality

Corresponding Author - Robert A. Semel, Ph.D., 1242 E. $70^{\text {th }}$ Street, 11234 Brooklyn, New York, United States, e-mail: robertsemelpsyd@gmail.com

AUthors' CONTRIBution - A: Study design - B: Data collection · C: Statistical analysis · D: Data interpretation · E: Manuscript preparation · F: Literature search · G: Funds collection

To CITE this ARTICLE - Semel, R. A. (2019). The Abbreviated Psychopathy Measure-2: associations with normal-range

and maladaptive personality traits. Current Issues in Personality Psychology, 7(2), 155-171.

RECEIVED 01.02.2019 · REVIEWED 08.04.2019 · ACCEPTED 06.05.2019 • PUBLISHED 28.05.2019 


\section{BACKGROUND}

In recent years, the study of psychopathic personality traits, which include interpersonal, affective, and behavioral features, such as manipulative and exploitive behavior, deceitfulness, superficial charm, callous lack of empathy, absence of guilt or remorse, egocentricity, impulsivity, and irresponsible lifestyle, has extended beyond offender samples to the broader population. This is in accordance with the increased consideration of psychopathic traits as being dimensional as opposed to categorical in nature, and as representing more extreme variants of normal personality traits. Research in psychopathy in non-forensic samples has been greatly expanding with the use of self-report measures of psychopathy. However, there is a lack of consensus in the field about how to conceptualize psychopathy, e.g., whether psychopathy includes positive adjustment indicators such as emotional stability, and how to measure or assess psychopathic traits, e.g., which latent dimensions underlie psychopathy. The Triarchic Psychopathy Measure (TriPM; Patrick, 2010) was developed from an organizing framework or integrative model of psychopathy (Patrick, Fowles, \& Krueger, 2009) that may help to reconcile different historical theories and contemporary measurement models through focusing on three distinct but intersecting phenotypic domains of Boldness, Meanness, and Disinhibition. These phenotypic components of psychopathy are conceptualized as dispositional tendencies associated with variations in the functioning of different biobehavioral systems (Drislane, Brislin, Jones, \& Patrick, 2018; Patrick, 2018; Patrick \& Drislane, 2014). The TriPM, a nonproprietary measure composed of 58 items, operationalizes these three domains.

As explicated by Patrick (2018), the triarchic model offers a contemporary explanation of the masked pathology paradox described by Cleckley (1941/1976), who described in certain patients the appearance of psychological normality, emotional stability, even what appeared as robust mental health, which co-existed with severe and persistent behavioral deviancy manifested by reckless, unrestrained behavior across multiple areas of life. The triarchic model posits a dualdisposition model in which two separate, uncorrelated dispositional tendencies co-occur, i.e., boldness and disinhibition. Boldness is associated with the biobehavioral concept of acute threat reactivity. Symptomatically or phenotypically, boldness manifests through personality qualities such as fearlessness, social dominance, low stress reactivity, emotional resiliency, and venturesomeness. Disinhibition is associated with the concept of inhibitory control which relates to distinct neurobiological systems. Phenotypically, disinhibition manifests through a propensity toward deficiencies in impulse control, including lack of planfulness and foresight, impaired regulation of affect and urges, demand for immediate gratification and deficient behavioral restraint. Meanness, or callous-unemotional tendencies, reflects a third dispositional tendency and relates to the concept of affiliation/attachment. Phenotypically, meanness manifests through a lack of empathy, disdain for and lack of close attachments with others, predatory exploitativeness, and empowerment through cruelty. As indicated by Patrick, meanness is believed to play a greater role in criminal expressions of psychopathy involving predatory exploitativeness and violence. Meanness and Disinhibition are conceptualized as moderately interrelated, Meanness and Boldness are conceptualized as more modestly interrelated, and Boldness and Disinhibition are conceptualized as minimally interrelated.

Drislane, Patrick, and Arsal (2014), Hall et al. (2014), as well as Patrick and Drislane (2014), contend that the triarchic domains may be conceptualized as "open constructs" that can be operationalized by different measures in differing ways. Several studies have demonstrated that reliable and valid triarchic scales can be developed by selecting relevant items from existing measures of psychopathy as well as from broad-band measures of personality and psychopathology, that capture the triarchic dimensions.

The TriPM and other triarchic scales that have been derived from existing measures of psychopathy, psychopathology, and personality have been found in community, undergraduate, and/or forensic samples to be significantly and differentially associated in expected directions with a wide range of normal range personality criterion variables and criterion variables representing more extreme variants of normal range personality traits, e.g., social potency, antagonism, temperament/affectivity, entitlement, narcissism, impulsiveness, wellbeing, social closeness, aggression, alienation, sensation seeking, stress reaction, responsibility, harm avoidance, anxiety, manipulativeness, disinhibition, as well as criterion variables including substance and alcohol use problems and antisocial behavior. Notably, the TriPM and triarchic scales developed from other existing measures have demonstrated convergence with the Psychopathy ChecklistRevised (PCL-R; Hare, 2003), despite their different methods of assessment (e.g., Brislin, Drislane, Smith, Edens, \& Patrick, 2015; Brislin et al., 2017; Hall et al., 2014; Murphy, Skeem, \& Edens, 2016; Venables, Hall, \& Patrick, 2014).

Recently, a new, abbreviated measure, based on the triarchic model but with different items, was developed by this author (Semel, 2018). This new measure, composed of 33 items and termed the Abbreviated Psychopathy Measure (APM), was developed not as a short form of the TriPM, but rather as an alternative, shorter operationalization of the triarchic model which may be more economical in some research contexts. In two pilot studies by Semel (2018), 
the total score of the APM was highly correlated with the TriPM Total score $(r=.90)$. Each APM scale was highly correlated with its counterpart scale on the TriPM. Each of the APM scales was also significantly correlated with a measure of Antisocial Intent. The APM Total score was also found to be highly correlated with the Total score of a 36-item version of the Levenson Self-Report Psychopathy Scale (LSRP; Levenson, Kiehl, \& Fitzpatrick, 1995) that was developed by Christian and Sellbom (2016). Further, the APM scales were associated differentially with Five-Factor Model (FFM) normal range personality dimensions associated with psychopathy (e.g., Boldness was robustly associated with Extraversion and had a modest, negative association with Neuroticism, Meanness was highly and inversely associated with Agreeableness, Disinhibition was robustly and negatively associated with Conscientiousness, and had a modest, positive association with Neuroticism).

Notwithstanding the initial encouraging findings with the APM, the correlational relationships among the APM scales differed significantly in comparison to the TriPM in that APM Boldness was moderately correlated with Meanness and Disinhibition. APM Meanness and Disinhibition were highly correlated with each other. The correlational configuration of the APM triarchic scales as found in the initial studies suggests that the APM might be most useful to study psychopathy as a higher-order construct containing correlated facets, as opposed to a configural construct containing facets that are more separate from one another (C. J. Patrick, personal communication, May 18, 2018). Furthermore, APM Boldness may capture a more maladaptive quality of boldness relative to TriPM Boldness through a greater emphasis on low harm avoidance, fearlessness, or a risky adventuresome propensity, and less emphasis on social efficacy and emotional resilience in comparison to TriPM Boldness. In this sense, the APM Boldness scale appears similar to the Boldness scale that was developed from items of the Youth Psychopathic Traits Inventory (YPI; Andershed, Kerr, Stattin, \& Levander, 2002) by Drislane et al. (2015) (C. J. Patrick, personal communication, May 18, 2018). Drislane et al. (2015) found that the YPI-triarchic Boldness scale was moderately correlated with both the YPI-tri Meanness and YPI-tri Disinhibition scales, which may be problematic given that the triarchic model of psychopathy views boldness and disinhibition as distinct constructs both phenotypically and with respect to neurobiological etiologies. Drislane et al. (2015) found the YPI-tri Boldness scale to be less distinctively associated with the boldness dimension of psychopathy as compared with both the TriPM boldness scale and a triarchic boldness scale developed from the Psychopathy Personality Inventory (PPI; Lilienfeld \& Andrews, 1996), although YPI-tri Meanness and Disinhibition were better separated relative to the TriPM and the PPI. Drislane et al. (2015), and Drislane, Brislin, Jones, and Patrick (2018) suggest that the YPI has less than adequate content coverage for the boldness dimension of psychopathy.

Pechorro and colleagues (see Pechorro, DeLisi, Alberto, Ray, \& Simoes, 2018; Pechorro, Simoes, Alberto, \& Ray, 2018) developed and studied the use of a 21-item YPI-triarchic measure across samples of incarcerated male youths, detained female youths, and community youths. CFA results in each study provided strong support for a three-factor inter-correlated model. The shortened triarchic YPI measure demonstrated adequate psychometric properties regarding internal consistency, convergent validity, discriminant validity, and criterion-related validity. However, in their studies the correlations between YPI Boldness and Disinhibition ranged between .44 and .60 , and the correlations between Boldness and Meanness ranged between .46 and .56. Such high levels of shared variance among these YPI-tri scales may in part account for the findings that YPI-tri Boldness had the strongest association (compared to YPI-tri Meanness and Disinhibition) with a measure of reactive and proactive aggression in detained female youths. The magnitude of association between YPI-tri Boldness and a measure of callous-unemotional (CU) traits was very similar to the associations between YPI-tri Meanness and YPI-tri Disinhibition with the measure of CU traits. YPI-tri Boldness had the strongest association with a conduct disorder diagnostic index in a sample of male incarcerated youths. In a sample of community youths, YPI-tri Boldness had the highest partial correlation with a measure of reactive and proactive aggression. Thus, it appears that the YPI triarchic scales have demonstrated less distinctive associations with criterion variables than would be expected according to the triarchic model, and in comparison with the triarchic scales of the TriPM. However, it should be noted that, like the finding by Drislane et al., in the studies by Pechorro, DeLisi, et al. (2018), and Pechorro, Simoes, et al. (2018), YPI-tri Meanness and Disinhibition had lower correlations, and thus were more distinct, relative to their association found in many studies with the TriPM.

Initial findings with the APM are similar to findings with the YPI triarchic measures, particularly with respect to the moderate correlations between APM Boldness with Meanness and Disinhibition. However, the strength of association between APM Meanness and Disinhibition was notably higher in comparison to studies with YPI triarchic scales. It is possible that APM Boldness, relative to TriPM Boldness, might reflect more grandiose, egocentric, and audacious qualities, and, as such, may capture a more maladaptive variant of boldness, but at the cost of less adequate coverage of stress resilience and social poise or equanimity conceptualized in the boldness 
construct. Furthermore, considering the degree of overlap between the triarchic constructs as measured by the APM, the opportunity for study of distinct phenotypic and neurobehavioral aspects of psychopathy with this measure is limited. Subsequently, a decision was made by this author to modify and update item content to develop more distinct, less converging triarchic scales, which would bring the APM more in alignment with the TriPM and would facilitate greater focus on external correlates of the APM triarchic scales, and potentially contribute to the neuroscience approach to understanding psychopathic personality disorder.

\section{PURPOSE OF CURRENT STUDY}

The APM, now in updated form (APM-2), is a brief measure of psychopathy based on the triarchic model which is potentially appealing as it is moderately economical in research contexts. As noted previously by Semel (2018), it will be important to determine whether the APM triarchic scales are associated with maladaptive personality traits that are within the psychopathy nomological network. A more nuanced examination of the relationship between the APM-2 scales and specific personality traits or facets may be helpful for this purpose. It is important to try to parse out whether APM-2 Boldness is associated with social efficacy and emotional stability as well as with a risk taking, adventure-seeking propensity. The TriPM Boldness scale and the Boldness scale developed from the Multidimensional Personality Questionnaire (MPQ; Tellegen, 1995/2003) by Brislin et al. (2015) have demonstrated significant associations with social potency and stress reaction (positively and negatively, respectively), as well as with sensation seeking. Does that hold true for APM-2 Boldness as well? While APM Meanness and Disinhibition were previously found to be inversely associated with FFM Agreeableness and Conscientiousness, respectively, are these triarchic scales associated at a trait or facet level with personality variables such as callousness, hostile aggression, lack of planning/impulsiveness, norm violation? The current study represents an effort to validate the APM-2 through study of its associations with normal-range personality variables and conceptually relevant maladaptive personality traits, and with a measure of antisocial intent, a correlate of antisocial behavior. Such a study may add to the growing literature and understanding of psychopathy in reference to underlying personality trait dimensions and maladaptive individual functioning. It is anticipated that the APM-2 Total score will be significantly associated with all the personality variables selected for study. It is anticipated that APM-2 Boldness will be positively associated with measures of power seeking and sensation seeking, while being inversely associated with a measure of emotional instability. It is anticipated that APM-2 Meanness will be associated with measures of callousness, manipulativeness, and hostile aggression, and with a measure of antisocial intent. It is anticipated that APM-2 Disinhibition will be positively associated with measures of norm violation, non-planfulness, impulsivity, sensation seeking, emotional instability/stress reaction, and antisocial intent.

\section{PARTICIPANTS AND PROCEDURE}

\section{PARTICIPANTS}

Participants $(N=208)$ were recruited through Amazon Mechanical Turk (MTurk), an online crowdsourcing platform in which "workers" perform $\mathrm{Hu}$ man Intelligence Tasks (HITS) for "requesters" for the completion of computerized tasks. MTurk has become increasingly popular as a resource for behavioral research data among social scientists (Paolacci \& Chandler, 2014). Burhmester, Kwang, and Gosling (2011) found MTurk participants to be slightly more demographically diverse in comparison to standard Internet samples and American college samples. Also, the data obtained from MTurk participants were at least as reliable as those obtained through traditional methods (see Miller, Crowe, Weiss, Lynam, \& Maples-Keller, 2017 for further discussion of strengths and potential limitations of using MTurk participants in personality disorder research.). In the current study, participants included 115 men (60\%) and 75 women $(40 \%)$ between the ages of 21 and 74 years, with a mean age of $36.9(S D=11.1)$.

\section{PROCEDURE}

Participants recruited from MTurk were directed to a link at SurveyMonkey where they were provided a brief description of the study and elements contained in the framework for informed consent, such as expected length of time, potential risks, discomforts, benefits, voluntary nature of the study, confidentiality, and the researcher's contact information. Participants provided their consent on the survey form prior to answering the remainder of the survey. Consent was recorded electronically together with the participants' survey responses. Participants were paid \$1.30 through MTurk for their participation. All participants received the same order of items.

Three validity items were embedded in the survey to assess whether participants were attending to the items. For one item, participants were directed to choose disagree strongly for that item. Participants were directed to choose very accurate and very inac- 
curate, respectively, for the successive two validity items. Participants' data were not included in analyses if they did not correctly respond to all three validity items. This resulted in the exclusion of 12 out of 208 participants (5.8\%). Additional participants were excluded for responding excessively quickly (completing the 136 questions in less than four minutes, which would indicate responding to one item in less than 1.7 seconds, on average). The average length of time for completion of this survey was 10 minutes. Seven participants were excluded on this basis, with one of these seven having already been excluded based on the validity items.

\section{MEASURES}

The Abbreviated Psychopathy Measure-Revised $(A P M-2)$ is a 33-item self-report scale designed to assess the constructs of Boldness, Meanness, and Disinhibition as postulated by Patrick et al. (2009). Item modifications or substitutions were made on each of the three scales with the aim of developing more distinctly representative measures of the respective constructs as described by Patrick et al. (2009), and by Patrick (2010) (see Appendix for a list of items). An effort was made to not overly saturate the Boldness scale with items which associate danger with excitement or fun, whereas risk and adventure seeking were already represented by other Boldness items. Three items that emphasized danger or were moderately to highly correlated with the Meanness or Disinhibition scales were eliminated (i.e., "Situations that others might see as dangerous I would see as exciting"; "What's the fun in life unless you're willing to face a little danger?"; "My fearless and stress-free personality would help me to be good at being an undercover agent or spy"). Those three items were replaced with items (all items were written by the author) intended to capture greater representation of social potency and stress resilience (i.e., "I approach life with a great sense of self-confidence"; "I sometimes have doubts about my ability to handle challenges that I face" (Reverse scored); "I am well able to manage even the most stressful situations"). Items on the APM Meanness and Disinhibition scales were eliminated or modified primarily based on high inter-item correlation (above $r=.60$ ) both within the respective scales and between the two scales. Three items on the APM-2 Meanness scale were modified but are similar in content to items on the APM (e.g., APM-2: "It would be hard for me to be cold and callous to other people's feelings"; APM: "It would be very hard for me to be cold and heartless to people"). The other eight items are new items. Four items on the APM-2 Disinhibition scale are similar in content to items on the APM Disinhibition scale (e.g., APM-2: "I tend to act quickly without thinking about the consequenc- es, and this sometimes gets me in trouble"; APM: “I'm the type of person to act quickly without thinking it through, which sometimes gets me in trouble"; APM-2: "I have impulsively stolen things"; APM: "I have stolen my share of things; the trick is just not to get caught”). The other seven items are new items. The response format for the APM-2 items continues to utilize a 4-point Likert-type scale that includes the choices disagree strongly, disagree somewhat, agree somewhat, agree strongly. Scores range from 1 to 4 . Higher scores reflect higher levels of their respective dimensions.

International Personality Item Pool-MPQ (IPIP-MPQ). The IPIP-MPQ, is a self-report questionnaire available in the public domain (Goldberg et al., 2006) composed of 12 preliminary IPIP scales measuring constructs similar to those in Tellegen's Multidimensional Personality Questionnaire (MPQ; Tellegen, 1995/2003). The current study included two IPIP-MPQ scales, i.e., Power-Seeking and Emotional Instability. The IPIP Power-Seeking scale (10 items; $\alpha=.85$ ) is a proxy measure for the MPQ Social Potency (SP) scale. MPQ Social Potency is one of four lower order scales that measure positive emotionality. It measures tendencies toward dominance, persuasiveness, and leadership. Those who score high on the SP scale describe themselves as forceful and decisive, persuasive and liking to influence others, enjoying leadership roles, liking to be noticed and the center of attention. IPIP Power-Seeking has a correlation of .80 with MPQ Social Potency (.92 when corrected for scale reliabilities). IPIP Emotional Instability (10 items; $\alpha=.84$ ) is a proxy measure for the MPQ Stress Reaction (SR) scale. MPQ Stress Reaction is a primary trait scale assessing negative emotional disposition. Those who score high on the SR scale describe themselves as prone to worrying and feeling anxious, tense, and nervous; sensitive and vulnerable; irritable and easily upset; having changing moods. IPIP Emotional Instability has a correlation of .75 with MPQ Stress Reaction (.87 when corrected for scale reliabilities).

International Personality Item Pool - Computerized Adaptive Test of Personality Disorders Static Form (CAT-PD-SF, v1.1). The CAT-PD-SF, v1.1, is an inventory based on the CAT-PD project (Simms et al., 2011) that was designed to develop a comprehensive and integrative set of higher and lower order personality traits relevant to personality pathology. Five CAT-PD-SF scales were included in the present study. Callousness ( 7 items; Community sample $\alpha=.85$; Patient sample $\alpha=.83$ ) reflects cold-heartedness, disregard for the rights, feelings, and welfare of others, and lack of sympathy and empathy. Hostile Aggression (8 items; Community sample $\alpha=.82$; $\mathrm{Pa}$ tient sample $\alpha=.87$ ) reflects a pattern of hostile and violent behavior that is either instrumental or reactive. The scale also taps the tendency to be resentful, mean-spirited, vindictive, and sadistic. Manipula- 
tiveness ( 6 items; Community sample $\alpha=.88$; Patient sample $\alpha=.85$ ) assesses a behavioral pattern of taking advantage of, and exploiting others to achieve self-serving goals, and a tendency to lie, cheat, and behave in overtly or covertly dishonest ways. NonPlanfulness (6 items; Community sample $\alpha=.82$; Patient sample $\alpha=.84)$ measures the tendency to act on whims or on the spur of the moment without planning or concern for the consequences. Norm Violation (7 items; Community sample $\alpha=.83$; $\mathrm{Pa}-$ tient sample $\alpha=.84$ ) reflects a general disregard for, and active rejection of social rules and conventions, a history of engaging in illegal or antisocial acts, and a pattern of disobedient and defiant behavior towards authority figures.

International Personality Item Pool - SensationSeeking. The IPIP Sensation-Seeking scale is a selfreport questionnaire composed of three IPIP sensation-seeking facet-level scales measuring constructs similar to those in the measure of sensation seeking by Hoyle, Stephenson, Palmgreen, Lorch, and Donohew (2002). Two of the three facet scales were included in the current study, i.e., Dangerous thrill-seeking (10 items; $\alpha=.86$ ) and Calculated thrill-seeking (10 items; $\alpha=.78$ ). Examples of the Dangerous thrillseeking scale include the items "Might actually enjoy being caught in an earthquake or tornado"; "Prefer fear to boredom". Examples of the Calculated thrillseeking scale include the items "Am willing to take risks"; "Would love to explore strange places".

Barratt Impulsiveness Scale-Brief Form (BIS-Brief). The Barratt Impulsiveness Scale, in its 11th edition (BIS-11; Patton, Stanford, \& Barratt (1995), is one of the most widely used measures of impulsivity. Steinberg, Sharp, Stanford, and Tharp (2013), using item response theory methods, developed a unidimensional short form containing eight of the original BIS-11 items. These eight items were identified by Steinberg et al. (2013) as efficient indicators of the underlying general impulsivity dimension. The BIS-Brief, like the BIS-11, is scored on a four-point scale ranging from 1 (rarely/never) to 4 (almost always/always).

The Measures of Criminal Attitudes and Associates (MCAA). The Measures of Criminal Attitudes and Associates (MCAA; Mills, Kroner, \& Forth, 2002) is a two-part self-report measure of antisocial attitudes and antisocial associates. The MCAA has demonstrated significant associations with other measures of antisocial attitudes, criterion validity in relation to criminal history indices, and has demonstrated predictive validity for the outcomes of general and violent recidivism in a sample of male adult offenders (Mills, Kroner, \& Hemmati, 2004). In the current study, only the 12-item Antisocial Intent scale was used, with response choices ranging from strongly disagree to strongly agree, on a 7-point Likert-type scale, to increase the variance at the extremes of the distribution.

\section{DATA ANALYSIS}

Descriptive statistics were examined for all study measures. To evaluate the construct validity of the APM-2 scales in relation to criterion variables, Pearson correlation coefficients were computed between the APM-2 scales and the criterion variables. In addition, to evaluate the unique contribution of each APM-2 scale to prediction of criterion variables after controlling for the other two APM-2 scales, multiple regression analyses were conducted in which all three APM-2 scales were entered simultaneously as predictors. To control for the number of analyses performed, conservative significance values of .005 (.05/11 criteria) were used. Quantitative analyses were conducted using SPSS Version 22 software.

\section{RESULTS}

Table 1 reports means, standard deviations, and internal consistencies for all study variables. All measures had adequate to high internal consistency coefficients (as measured by Cronbach's $\alpha$ ). Mean inter-item correlations for each of the APM-2 triarchic scales and for the APM-2 Total scale were within the range of .15 to .50 , indicating an acceptable level of internal consistency (Clark \& Watson, 1995). Internal consistency coefficients were very similar for men and women. Men scored significantly higher on the Boldness and Meanness scales and on the APM-2 Total score. Effect sizes (Cohen's d) were 0.44, 0.38, and 0.34 , respectively, reflecting small to medium effect size differences. There was no significant mean score difference by gender on the Disinhibition scale $(d=-0.06)$, which is not a unique finding for triarchic disinhibition (see Discussion). With respect to criterion variables, $t$-tests indicated that men obtained significantly higher mean scores on Dangerous thrill-seeking $(t(188)=2.95, p=.004)$, Calculated thrill-seeking $(t(188)=4.03, p<.001)$, and Antisocial Intent $(t(188)=2.52, p=.013)$, whereas women obtained a significantly higher mean score on Emotional Instability $(t(188)=3.50, p<.001)$. Age was significantly and negatively associated with all the APM-2 scales. Effect sizes (coefficient of determination) were small for the three individual scales and for the APM-2 Total score - the variance explained by age was less than $9 \%$ for each of the scales. Intercorrelations among the three APM-2 scales were as follows: Boldness and Meanness, $r=.34(p<.001)$; Boldness and Disinhibition, $r=.09$ (ns); Meanness and Disinhibition, $r=.61(p<.001)$. In comparison to the original APM, there was better distinction among the APM-2 triarchic scales; however, the association between Meanness and Disinhibition remained high in the current sample. The intercorrelations among the APM-2 scales were similar for men and women. 
Table 1

Means (M), standard deviations (SD) and internal consistency coefficients of all measures

\begin{tabular}{lccc}
\hline Scale & $M$ & $S D$ & $\alpha$ \\
\hline APM-2 Boldness & 26.03 & 6.75 & .86 \\
APM-2 Meanness & 18.66 & 5.81 & .84 \\
APM-2 Disinhibition & 19.97 & 6.34 & .86 \\
APM-2 Total & 64.67 & 14.10 & .89 \\
Power Seeking & 28.76 & 9.19 & .90 \\
Emotional Instability & 25.52 & 10.35 & .93 \\
Callousness & 12.24 & 6.22 & .93 \\
Hostile Aggression & 12.26 & 6.50 & .93 \\
Manipulativeness & 10.19 & 5.22 & .90 \\
Non-Planfulness & 11.62 & 5.15 & .88 \\
Norm Violation & 13.15 & 5.96 & .86 \\
Sensation Seeking Danger & 19.88 & 8.91 & .89 \\
Sensation Seeking Calculated & 30.11 & 8.00 & .82 \\
BIS Brief & 13.38 & 4.23 & .84 \\
Antisocial Intent & 34.52 & 15.63 & .89 \\
\hline
\end{tabular}

Note. APM-2 - Abbreviated Psychopathy Measure-2; Power Seeking and Emotional Stability are from the International Personality Item Pool (IPIP) proxy measure for the Multidimensional Personality Questionnaire; Callousness, Hostile Aggression, Manipulativeness, Non-Planfulness, Norm Violation are from the IPIP Computerized Adaptive Test of Personality Disorders Static Form (CAT-PD-SF); Sensation Seeking Danger and Sensation Seeking Calculated scales are from the IPIP Sensation Seeking scale; BIS Brief - Barratt Impulsiveness Scale-Brief Form; Antisocial Intent is from the Measures of Criminal Attitudes and Associates (MCAA).

The largest gender difference between correlation coefficients was for the inter-correlation between Meanness and Disinhibition, $r=.66, p<.001$, and $r=.56, p<.001$, for men and women, respectively. However, when the difference between these correlations was examined by transforming correlations into $z$-scores using Fisher's $r$-to- $z$ transformation, the correlation coefficients between Meanness and Disinhibition for men and women were not significantly different (Fisher's $Z=1.06, p=.145$ ).

\section{CRITERION-RELATED VALIDITY OF APM-2 SCALES}

Zero-order correlations are reported in Table 2 between APM-2 scales and criterion measures, along with standardized $\beta$ coefficients from multiple regression analyses using the three APM-2 scales as concurrent predictors of each criterion measure in order to examine the unique contribution of each APM-2 scale prediction. The correlations between the APM-2 Total score and each criterion variable are also shown.

APM-2 Total scores demonstrated significant, moderate to strong correlations with all criterion variables except for Emotional Instability (see Discus- sion section). As would be expected of a psychopathy measure, APM-2 Total score was highly associated with measures of Callousness, Hostile Aggression, Manipulativeness, Non-Planfulness, Sensation Seeking (of a dangerous thrill-seeking quality), Norm Violation, and with Antisocial Intent. The associations between each of the APM-2 scales and the criterion variables will be discussed separately.

Boldness. As anticipated, APM-2 Boldness demonstrated a quite robust association with IPIP Power Seeking (proxy for MPQ Social Potency), both at the bivariate level and in regression analyses. APM-2 Boldness was the only APM-2 scale that had a significant zero-order correlation with Power Seeking and was also a unique predictor of this criterion variable. APM-2 Boldness, as anticipated, was significantly and negatively associated with Emotional Instability (proxy for MPQ Stress Reaction) at the zero-order level and had a strong unique contribution to scores on Emotional Instability in a regression analysis. As anticipated, Boldness was significantly associated with Sensation Seeking, both with the Dangerous thrillseeking scale and the Calculated thrill-seeking scale, both at the bivariate level and in regression analyses. In the regression analysis, only Boldness emerged as a unique predictor of Calculated thrill-seeking. 
Table 2

Zero-order correlations and multiple regression betas for APM-2 scales as predictors of psychopathy-relevant criterion measures

\begin{tabular}{|c|c|c|c|c|c|}
\hline Criterion measures & Total & Boldness, $r / \beta$ & Meanness, $r / \beta$ & Disinhibition, $r / \beta$ & $R^{2}$ \\
\hline Power Seeking & $.42^{*}$ & $.76^{*} / .78^{*}$ & $.17 /-.05$ & $-.03 /-.08$ & $.59^{*}$ \\
\hline Emotional Instability & .03 & $-.50 * /-.55^{*}$ & $.14 / .01$ & $.48^{*} / .52 *$ & $.53^{*}$ \\
\hline Callousness & $.61^{*}$ & $.15 /-.08$ & $.70^{*} / .63^{*}$ & $.54 * / .17$ & $.52^{*}$ \\
\hline Hostile Aggression & $.74^{*}$ & $.27 * / .05$ & $.77^{*} / .57^{*}$ & $.65 * / .30 *$ & $.64^{*}$ \\
\hline Manipulativeness & $.71^{*}$ & $.23 * / .01$ & $.75^{*} / .56^{*}$ & $.65 * / .31 *$ & $.62^{*}$ \\
\hline Non-Planfulness & $.59^{*}$ & $.09 / .01$ & $.48 * / .01$ & $.78^{*} / .77^{*}$ & $.60^{*}$ \\
\hline Norm Violation & $.71^{*}$ & $.30 * / .21^{*}$ & $.57 * / .10$ & $.73^{*} / .65^{*}$ & $.60^{*}$ \\
\hline SS Danger & $.68^{*}$ & $.52 * / .42^{*}$ & $.54 * / .22 *$ & $.46^{*} / .29^{*}$ & $.47^{*}$ \\
\hline SS Calculated & $.38^{*}$ & $.63^{*} / 64^{*}$ & $.17 /-.02$ & $.01 /-.03$ & $.46^{*}$ \\
\hline BIS Brief & $.35^{*}$ & $-.19 /-.24^{*}$ & $.31 * /-.09$ & $.71^{*} / .79^{*}$ & $.58^{*}$ \\
\hline Anti-Social Intent & $.65^{*}$ & $.19 / .03$ & $.64^{*} / .36^{*}$ & $.67^{*} / .45^{*}$ & $.53^{*}$ \\
\hline
\end{tabular}

Note. Zero-order correlation coefficients and standardized beta-weights in boldface are of at least moderate effect size (.30); SS Danger - Dangerous thrill-seeking scale; SS Calculated - Calculated thrill-seeking scale; BIS Brief - Barratt Impulsiveness Scale-Brief Form;

Antisocial Intent is from the Measures of Criminal Attitudes and Associates (MCAA); ${ }^{*} p<.005$.

Boldness was marginally and modestly associated negatively with a measure of impulsiveness. The relationship between Boldness and Impulsiveness remained modest but was significant, inversely, as a unique predictor when all APM-2 scales were examined as concurrent predictors in a regression analysis.

Boldness had significant, positive, albeit small to medium associations with Hostile Aggression, Manipulativeness, and Norm Violation. However, after controlling via regression analyses for variance shared with the other two APM-2 scales, the associations between Boldness and Hostile Aggression and Manipulativeness no longer remained significant. In contrast, Boldness evidenced significant positive associations with Norm Violation both at the bivariate level and in regression analyses, although the associations were modest. Still, this is an interesting finding which will be further addressed in the discussion section.

Boldness was marginally associated with Antisocial Intent at the bivariate level, with a correlation $(r=.19, p=.008)$ that only slightly exceeded the threshold criterion for significance. However, in regression analysis, Boldness was not a significant predictor of Antisocial Intent $(\beta=.03)$. This was in accordance with our expectations.

Meanness. As expected, APM-2 Meanness was highly correlated with personality measures of Callousness, Hostile Aggression, Manipulativeness, and with Antisocial Intent, a correlate of antisocial behavior, suggesting that APM-2 Meanness may tap into essential affective, interpersonal, and antisocial qualities associated with psychopathy. Although Disinhibition also was significantly associated with Callousness, Hostile Aggression, and Manipulativeness, and remained a significant unique predictor of Hostile Aggression and Manipulativeness when entered concurrently in a regression model, Meanness was a stronger predictor. Meanness also was found to be significantly associated with Non-Planfulness, Norm Violation, and Impulsiveness at the zero-order level. However, Meanness was not found to be a unique predictor of these measures when all three APM-2 scales were entered concurrently in a regression model. Meanness also was significantly associated with Sensation-Seeking: Dangerous at the bivariate level and as a unique predictor in a regression model. Although this was not predicted, other studies have also found significant associations between TriPM Meanness and thrill or sensation seeking, as will be discussed later.

Disinhibition. Although both Disinhibition and Meanness were significantly associated with NonPlanfulness at the zero-order level, only Disinhibition emerged as a unique predictor when all APM-2 scales were examined as concurrent predictors in a regression analysis. All three APM-2 scales were significantly associated with Norm Violation at the zeroorder level. However, Disinhibition was the strongest unique predictor of Norm Violation $(\beta=.65)$. Predictions of both Meanness and Disinhibition to Hostile Aggression and Manipulativeness were reduced in the regression model, indicating overlap between Meanness and Disinhibition in predicting these per- 
sonality variables. As anticipated, Disinhibition was significantly associated with Sensation Seeking, but only with the Dangerous thrill-seeking scale. In a regression analysis, Disinhibition remained a unique, albeit modest predictor of Dangerous thrill-seeking. As anticipated, Disinhibition was found to be a robust predictor of impulsiveness, both at the zeroorder level, and as a unique predictor in a regression analysis. As expected, APM-2 Disinhibition was positively associated with Emotional Instability, the IPIP proxy scale for the MPQ Stress Reaction scale, at both the zero-order level and as a robust, unique predictor in a regression analysis $(\beta=.52)$. Finally, as anticipated, Disinhibition was a robust predictor of Antisocial Intent in both zero-order analyses and in a regression analysis.

Correlations between the APM-2 scales with the above criterion variables were generally similar for men and women, with few differences observed at the conservative significance value of .005 . The correlation coefficients between APM-2 Total and impulsivity as indexed by the BIS Brief scale were significantly different for men and women $(r=.57$, and $r=.17$, respectively; Fisher's $Z=3.15, p=.001)$. The association between APM-2 Meanness and the BISBrief scale was significantly stronger for men than for women ( $r=.53$, and $r=.13$, respectively; Fisher's $Z=2.98, p=.001)$. Several other correlation differences by gender would be considered significant using less stringent significance levels; for example, the difference between APM-Boldness and BIS-Brief (Fisher's $Z=2.07$ ) would be considered significant using an alpha level of $p<.05$; the correlation between Boldness and BIS-Brief was higher in magnitude, inversely, for women than for men.

Given the potential problem of common method variance resulting from the use of a single method of data collection which may influence the relationships between measures, a post hoc statistical analytical technique, i.e., Harman's single-factor test, was used to estimate the degree to which the data may be influenced by common method variance. Harman's single-factor technique (Podsakoff, MacKenzie, Lee, \& Podsakoff, 2003) uses an exploratory factor analysis in which all the study variables are loaded onto a single factor and the unrotated factor solution is examined. If the newly introduced common latent factor explains more than $50 \%$ of the variance (this is the customary heuristic to set for the threshold), then common method bias may be present. In the current case, the results of Harman's single-factor technique indicated that the total variance for the single factor was $29.98 \%$, less than the heuristic $50 \%$ threshold, which, of itself, does not indicate common method bias. It is acknowledged that there are multiple limitations of this procedure, including that it does not control for (or partial out) method effects (Podsakoff et al., 2003).

\section{DISCUSSION}

The primary aims of the current study were to validate an updated version of the Abbreviated Psychopathy Measure by improving its indexing of the triarchic scales through item alterations, and by examining its relationships with lower-order personality traits associated with psychopathy. Results of the current study complement and extend the initial two studies of the APM, which found that the APM was highly correlated with the TriPM, and that APM scales were differentially associated with personality dimensions from the Five-Factor Model of personality. In the current study, the APM-2 total score was found to be associated with fundamental, if not prototypical features within the psychopathy nomological network, including a socially confident, dominant, persuasive interpersonal style, in conjunction with a manipulative/exploitive, callous character with potential toward instrumental or reactively hostile and aggressive tendencies, and given toward rash, impulsive, sensation seeking tendencies, with disregard for social rules and conventions and a propensity toward engaging in illegal or antisocial acts. The APM-2 total score had no correlation with a measure of Emotional Instability (Stress Reaction). However, this finding is consistent with the findings of others, e.g., Drislane et al. (2014), who similarly found the TriPM Total score to have a zero-order correlation of .03 with the Stress Reaction scale of a short version of the MPQ. Also, Strickland, Drislane, Lucy, Krueger, and Patrick (2013) found the TriPM Total score to have a nonsignificant association with a measure of negative affect. Such findings are not unexpected given that it is particularly the externalizing/disinhibited dimension of psychopathy that is associated with negative emotionality, whereas Boldness is associated with suppression of internalizing/neurotic tendencies (Patrick, 2018). Scores on Boldness and Disinhibition might counterweigh one another in relation to Emotional Instability.

The current study found that despite the continued high inter-scale correlations on the updated APM, particularly between Meanness and Disinhibition, each APM-2 scale had unique or stronger associations with criterion personality variables than did the other two APM-2 scales in a manner consistent with the triarchic model. The findings and implications for each of the APM scales will be discussed in greater detail.

APM-2 Boldness was robustly associated with a proxy measure of social potency. This is a significant finding given that APM Boldness may capture a more maladaptive quality of boldness in comparison to TriPM Boldness, although the modest change in item composition on the updated measure may have mitigated sensitivity to some maladaptive correlates by reducing overlap of APM-2 Boldness with 
Meanness and with Disinhibition. At the same time, APM-2 Boldness had a strong unique relationship, inversely, with IPIP Emotional Instability. This may suggest that boldness, as captured by the APM-2, may index integral features as conceptualized in the triarchic model, i.e., social dominance, low stress reactivity, emotional resiliency.

As expected, APM-2 Boldness was robustly associated, both in zero-order correlations and in regression analyses, with two scale measures of sensation seeking. Interestingly, only APM-2 Boldness was uniquely associated with the Sensation Seeking Calculated thrill-seeking scale. Weidacker, O'Farrell, Gray, Johnston, and Snowden (2017), who studied the relationship of the triarchic psychopathy model to impulsivity in offenders and community participants, as measured by the UPPS-P (Lynam, Smith, Whiteside, \& Cyders, 2006), suggested that TriPM Boldness is associated with people who thrive on risk-taking situations and can take calculated risks while being calm and rational and able to plan ahead. In the current study, APM-2 Boldness appeared to be distinguished from both Meanness and Disinhibition with respect to calculated risk taking. APM-2 Boldness was associated with both Dangerous thrill-seeking and Calculated thrill-seeking, while also being negatively associated with impulsiveness. This configuration of APM-2 Boldness with risk taking and impulsiveness, positively and negatively, respectively, suggests similarities to the finding of Weidacker et al. (2017) in that people who are higher on the dimension of boldness may take more risks than others, including some risks that entail greater danger or potential loss, yet they are less inclined to be impulsive, they can remain calm, and they can plan ahead. This APM-2 Boldness configuration differed sharply from that of Disinhibition, which was moderately associated with Dangerous thrill-seeking, not associated with calculated thrillseeking, but strongly associated with impulsiveness. This is a very significant distinction between the Boldness and Disinhibition dimensions as indexed by the APM-2.

The current study suggests that boldness, as represented by items of the APM-2, may have modest associations with indicators of hostile/aggressive and manipulative/exploitive qualities, and even antisocial tendencies; however, such relationships were in large measure accounted for by meanness or disinhibition. Nevertheless, the finding that APM-2 Boldness was associated with both positive adjustment (social potency, emotional stability) and negative adjustment (both dangerous and more calculated sensation seeking in zero-order and regression analyses, and interpersonal, externalizing tendencies in zero-order correlational analyses) is consistent with various studies of the TriPM and triarchic scales developed from other measures.
Overall, APM-2 Boldness appears to be associated with a personality style that is dominant and persuasive, with an elevated sense of self-worth, emotionally stable, with an inclination toward venturesomeness, but with lower tendencies toward impulsiveness, and with somewhat manipulative and potentially hostile features. Whether APM-2 Boldness may operate as effectively as TriPM Boldness with respect to associations with negative outcomes remains to be seen. It would need to be determined in future research whether APM-2 Boldness would significantly predict scores on Factor 1 , and the Interpersonal facet, and Factor 2 of the PCL-R.

An interesting finding in the current study was that Boldness remained a significant, albeit weak or modest predictor of Norm Violation, while Disinhibition was a very robust predictor of Norm Violation. Speculatively, this finding may illustrate Patrick's (2018) assertion that one may find greater tendencies toward impulsive-antisocial behavior, as well as substance abuse, in persons high in disinhibition and in boldness. APM-2 Boldness may be sensitive to detecting a latent, synergistic interaction with disinhibition that augments potential for antisocial behavior.

APM-2 Meanness was found to be strongly and preferentially correlated with measures assessing lack of care, concern, and empathy for others, hostile, aggressive, vengeful qualities, and manipulative/ exploitative qualities, and antisocial intent. As such, APM-2 Meanness was effective in this study at assessing essential features associated with the construct of triarchic meanness (Patrick, 2010, 2018; Patrick et al., 2009). APM-2 Meanness may be able to index an affective dimension of psychopathy, while also being associated with an antisocial dimension, and, to a lesser extent, an interpersonal dimension of psychopathy, particularly with respect to manipulativeness/exploitativeness. In a study sample of forensic participants, Hall et al. (2014) found PPI-triarchic Meanness to be a significant predictor of both PCL-R Total score and Factor 1 and Factor 2 scores. PPI-Meanness was the strongest predictor of the Affective facet, and betas were also significant predictors of the Lifestyle and Antisocial facets. Similarly, Brislin et al. (2015) found MPQ-triarchic Meanness to be a significant predictor of PCL-R Total score, Factor 1, Factor 2, the Affective and the Antisocial facets in a sample of incarcerated male participants. Whether this might hold true for APM-2 Meanness remains to be seen. The current finding is also consistent with the previous finding (Semel, 2018) that APM Meanness was strongly associated (negatively) with Agreeableness as assessed with the Big Five Inventory (BFI; John, Donahue, $\&$ Kentle, 1991). Very low agreeableness/very high antagonism is considered by some (see Miller \& Lynam, 2015) to be most essential to the construct of psychopathy. Similar to the finding by Semel (2018), Hall et al. (2014) found that PPI-Meanness was a very robust 
predictor of NEO-PI-R Antagonism (Costa \& McCrae, 1992), both in bivariate and regression analyses. It is not known whether APM-2 Meanness would be a significant predictor of antisocial personality disorder and child conduct disorder symptoms as found with MPQ and PPI triarchic Meanness scales (Brislin et al., 2015; Brislin et al., 2017; Hall et al., 2014).

The association of APM-2 Meanness with dangerous thrill-seeking as seen in this study is consistent with findings that the MMPI-2-RF (Ben-Porath \& Tellgen, 2008) triarchic Meanness scale was significantly associated with a measure of risk taking (Kutchen et al., 2017), and MPQ-based Meanness was significantly associated with a sensation seeking scale (Brislin et al., 2017).

APM-2 Disinhibition appeared to be effective in assessing externalizing, rash, impulsive, risk taking, and rule or law-breaking tendencies, and, to a lesser extent, aggressive tendencies. As such, APM-2 Disinhibition may be considerably and meaningfully connected with lifestyle and antisocial facets of psychopathy as conceptualized in the PCL-R. At the same time, APM-2 Disinhibition was positively and significantly associated with internalizing, negative emotionality as assessed by a measure of Emotional Instability, the IPIP proxy measure of MPQ Stress Reaction, which is a primary trait scale assessing negative emotional disposition. This pattern of findings with APM-2 Disinhibition is quite consistent with findings for the TriPM Disinhibition scale and triarchic disinhibition scales as indexed by other measures (e.g., Brislin et al., 2015; Brislin et al., 2017; Drislane et al., 2014; Sellbom et al., 2016; Stanley, Wygant, \& Sellbom, 2013). Thus, APM-2 Disinhibition appears to be a potentially effective measure of impulsive/ disinhibitory tendencies along with negative emotionality as described by Patrick (2018).

Although APM-2 Disinhibition also was a unique predictor of Hostile Aggression, Meanness was the strongest predictor. Hall et al. (2014) found that PPIMeanness and PPI-Disinhibition contributed equally to the prediction of MPQ Aggression in a regression analysis. Kutchen et al. (2017) found the MMPI-2-RF Disinhibition scale, along with the Meanness scale, to be a significant predictor of measures of aggression. Further, Patrick (2018) notes, albeit with respect to violent offenders, that some persons display aggressive tendencies mostly as an expression of anger and weak restraint associated with disinhibition, while others display aggressive behavior primarily resulting from lack of sensitivity, a low level of social concern, and predatory goal seeking associated with meanness. An insight by Tapscott, Vernon, and Veselka (2012), concerning the moderate to strong association between PCL-R Factor 1 and Factor 2, might be relevant here. That is, Tapscott et al. (2012) noted that low Agreeableness is represented in the items of both PCL-R factors. Hence, the two factors correlate, and aggres- sion may be a manifestation of both PCL-R factors, and of triarchic meanness and disinhibition.

The finding that APM-2 Disinhibition remained a significant predictor of manipulativeness in a regression analysis may be similar to the finding by Brislin et al. (2015) in which MPQ-based Disinhibition had the strongest contribution to the NPI Exploitativeness scale. MPQ-based Disinhibition and Meanness were similarly predictive of Exploitativeness as there was overlap between the two scales.

Men in this study obtained significantly higher mean scores than women on APM-2 Boldness, Meanness, and APM-2 Total score, with small to medium effect size differences. The absence of gender difference on the Disinhibition scale is interesting, although not unique. Brislin et al. (2017) similarly found that male undergraduates scored significantly higher than females on MPQ Boldness and Meanness, but not Disinhibition, whereas male offenders scored higher than female offenders on all three MPQ triarchic scales. Drislane and Patrick (2017) also found that a sample of male and female undergraduates did not differ in mean scores on TriPM Disinhibition. Drislane and Patrick speculated that manifest indicators of disinhibition in their latent variable model of triarchic psychopathy constructs may have assessed unconstrained/uninhibited tendencies more in traitdispositional terms rather than through reference to more explicit deviant behavior. This consideration might apply as well with respect to the APM-2 Disinhibition scale. Although the APM-2 Disinhibition scale, like the TriPM Disinhibition scale, includes some items that refer to antisocial behavior, it is possible that both scales are effective in capturing personality traits associated with manifest expressions of disinhibition, rather than primarily tapping into externalizing behavior. It has been noted (see Skeem, Polaschek, Patrick, \& Lilienfeld, 2011, for a review of psychopathic personality) that gender differences may be expressed differentially through greater proneness to externalizing behaviors in men and internalizing problems in women. Poy, Segarra, Esteller, Lopez, and Molto (2014) found that men scored higher than women on all three TriPM triarchic scales; however, women scored higher than men on a measure of FFM Neuroticism. In the current study, men obtained significantly higher mean scores on externalizing criterion variables, particularly sensation/thrill seeking and antisocial intent, whereas women obtained a significantly higher mean score on a criterion variable reflecting internalizing problem, i.e., emotional instability. The current findings may be consistent with the literature on gender differences in psychopathy (Skeem et al., 2011). However, further study of possible gender differences on the APM-2 and in relation to external correlates would be helpful.

Revisions made in the APM-2 did operate to reduce inter-scale correlations, although this study 
found that meanness and disinhibition were still highly correlated. This is not desirable from a conceptual and measurement perspective (e.g., the possibility of multicollinearity resulting in increases in standard errors and regression coefficients being incorrectly estimated). The degree of collinearity in the current study might be at least partly sample specific. In a previous study using the original APM (Semel, 2018), and which also included the TriPM, meanness and disinhibition had a correlation of .74 on both the APM and the TriPM. Poy et al. (2014) reported a correlation of $r=.62$ for TriPM Meanness and Disinhibition. Fanti, Kyranides, Drislane, Colins, and Andershed (2016) reported a correlation of .59 between Meanness and Disinhibition. Kyranides, Fanti, Sikki, and Patrick (2017) reported a correlation of .66 between Meanness and Disinhibition. Thus, the correlation of .61 between APM-2 Meanness and Disinhibition in the current study is not unique and is within the range of correlations between TriPM Meanness and Disinhibition, i.e., . 4 to .6 reported across various studies. However, most other studies have reported more moderate relationships between triarchic meanness and disinhibition. It remains to be seen whether the correlation between APM-2 Meanness and Disinhibition might be lower in other samples.

\section{LIMITATIONS AND FUTURE DIRECTIONS FOR RESEARCH}

A limitation of the current study is that all study variables were assessed by self-report questionnaires, which increases the possibility for artificially inflating correlations among the study measures due to monomethod bias. Although a post hoc analysis (Harman's single-method technique) did not indicate common method bias, future studies should include assessment of psychopathy and psychopathy-related personality traits through additional methods (e.g., PCL-R, and interview-based DSM assessment, in conjunction with self-report measures). Additional procedural remedies, as suggested by Podsakoff et al. (2003), should also be considered, e.g., temporal, proximal, or methodological separation of measurement of the independent and dependent variables, counterbalancing question order, improving scale items, as well as protecting respondent anonymity (anonymity and confidentiality were maintained in this study). Also, replication and extension of study of the APM-2 to include a greater range of external correlates, including personality and behavioral variables, is needed to determine whether the nomological networks of the APM-2 scales would make the APM-2 useful as a shorter, alternative triarchic psychopathy measure.

Participants were recruited from MTurk, which limits generalizability to other settings. Berinski, $\mathrm{Hu}$ ber, and Lenz (2012) found that MTurk workers were notably younger and more ideologically liberal than the general public, they appear more attentive to tasks, but they may also exhibit experimental demand characteristics to a greater degree than do respondents in other subject pools, and habitual responding may pose more of an external validity problem. Overall, studies have found support for the reliability and validity of data collected on MTurk for personality disorder research and in conducting research on clinical populations (see Miller et al., 2017; Shapiro, Chandler, \& Mueller, 2013). Nevertheless, it would be beneficial to extend study of the APM-2 to other populations and settings, including forensic settings. Limited demographic data were obtained, and future research should include expanded demographic data.

The primary advantages of shorter psychopathy measures include the fact that they may be more convenient to access large numbers of persons, which is of benefit in epidemiological research, they may be useful in research contexts in which there are significant time constraints, and they may be used for screening purposes (Eisenbarth, Lilienfeld, \& Yarkoni, 2015; Tonnaer, Cima, Sijtsma, Uzieblo, \& Lilienfeld, 2013). This is evident in the development of short forms of other inventories such as the Psychopathic Personality Inventory-Revised (PPI-R; Lilienfeld \& Widows, 2005) and the Elementary Psychopathy Assessment (EPA; Lynam et al., 2011). Whether the APM-2 adequately reflects the range or breadth of psychopathy-related personality traits as indexed by the TriPM, and whether it might be useful in basic research, remains to be seen.

Notwithstanding potential advantages of the APM-2 as noted above, the TriPM is a well-validated measure, and psychopathy-relevant variables can be studied from existing archival databases in which triarchic scales can now be extracted from existing measures such as the PPI-R, MPQ, MMPI-2-RF, and the NEO-PI-R. This is a major advantage over any new self-report psychopathy measure that may be introduced. Still, there is a continual need for study of psychopathy in new samples with respect to the complex, multiple causal pathways, and variants of psychopathy (e.g., biobehavioral, developmental, environmental factors), and implications for prevention and for intervention/treatment considerations. The APM-2 might contribute to such research.

\section{REFERENCES}

Andershed, H., Kerr, M., Stattin, H., \& Levander, S. (2002). Psychopathic traits in non-referred youths: A new assessment tool. In E. Blaauw \& L. Sheridan (Eds.), Psychopaths: Current international perspectives (pp. 131-158). The Hague: Elsevier.

Ben-Porath, Y. S., \& Tellegen, A. (2008). Minnesota Multiphasic Personality Inventory-2 Restructured 
Form: Manual for administration, scoring, and interpretation. Minneapolis, MN: University of Minnesota Press.

Berinski, A. J., Huber, G. A., \& Lenz, G. S. (2012). Evaluating online labor markets for experimental research: Amazon.com's Mechnical Turk. Political Analysis, 20, 351-368. http://dx.doi.org/10.1093/ pan/mpr057

Brislin, S. J., Drislane, L. E., Smith, S. T., Edens, J. F., \& Patrick, C. J. (2015). Development and validation of triarchic psychopathy scales from the Multidimensional Personality Questionnaire. Psychological Assessment 27, 838-851. http://dx.doi. org/10.1037/pas000087

Brislin, S. J., Venables, N. C., Drislane, L. E., Blonigen, D. M., Iacono, W. G., Tellegen, A., Edens, J. F., \& Patrick, C. J. (2017). Further validation of triarchic psychopathy scales from the Multidimensional Personality Questionnaire: Setting the stage for large-sample etiological studies. Assessment, 24, 575-590. https://doi.org/10.1177/1073191115621790

Burhmester, M., Kwang, T., \& Gosling, S. D. (2011). Amazon's Mechanical Turk: A new source of inexpensive, yet high-quality, data? Perspectives on Psychological Science, 6, 3-5. http://dx.doi.org/ $10.1177 / 1745691610393980$

Christian, E., \& Sellbom, M. (2016). Development and validation of an expanded version of the threefactor Levenson Self-Report Psychopathy Scale. Journal of Personality Assessment, 98, 155-168. https://doi.org/10.1080/00223891.2015.1068176

Clark, L. A., \& Watson, D. (1995). Constructing validity: Basic issues in objective scale development. Psychological Assessment, 7, 309-319. http://dx.doi. org/10.1037/1040-3590.7.3.309

Cleckley, H. (1941). The mask of sanity. St. Louis, MO: Mosby.

Cleckley, H. (1976). The mask of sanity (5 ${ }^{\text {th }}$ ed.). St. Louis, MO: Mosby.

Costa, P. T., Jr., \& McCrae, R. R. (1992). Revised NEO Personality Inventory (NEO-PI-R) and NEO FiveFactor Inventory (NEO-FFI) professional manual. Odessa, FL: Psychological Assessment Resources.

Drislane, L. E., Brislin, S. J., Jones, S., \& Patrick, C. J. (2018). Interfacing five-factor model and triarchic conceptualizations of psychopathy. Psychological Assessment, 30, 834-840. http://dx.doi.org/10.1037/ pas0000544

Drislane, L. E., Brislin, S. J., Kendler, K. S., Andershed, H., Larsson, H., \& Patrick, C. J. (2015). A triarchic model analysis of the Youth Psychopathic Traits Inventory. Journal of Personality Disorders, 29, 15-41. https://doi.org/10.1521/pedi_2014_28_144

Drislane, L. E., \& Patrick, C. J. (2017). Integrating alternative conceptions of psychopathic personality: A latent variable model of triarchic psychopathy constructs. Journal of Personality Disorders, 31, 110-132.
Drislane, L. E., Patrick, C. J., \& Arsal, G. (2014). Clarifying the content coverage of differing psychopathy inventories through reference to the Triarchic Psychopathy Measure. Psychological Assessment, 26, 350-362. http://dx.doi.org/10.1037/a0035152

Eisenbarth, H., Lilienfeld, S. O., \& Yarkoni, T. (2015). Using a genetic algorithm to abbreviate the Psychopathic Personality Inventory-Revised (PPIR). Psychological Assessment, 27, 194-202. http:// dx.doi.org/10.1037/pas0000035

Fanti, K. A., Kyranides, M. N., Drislane, L. E., Colins, O. F., \& Andershed, H. (2016). Validation of the Greek Cypriot translation of the Triarchic Psychopathy Measure. Journal of Personality Assessment, 98, 146-154. http://dx.doi.org/10.1080/00223 891.2015.1077452

Goldberg, L. R., Johnson, J. A., Eber, H. W., Hogan, R., Ashton, M. C., Cloninger, C. R., \& Gough, H. C. (2006). The International Personality Item Pool and the future of public-domain personality measures. Journal of Research in Personality, 40, 84-96. http://dx.doi.org/10.1016/j.jrp.2005.08.007

Hall, J. R., Drislane, L. E., Patrick, C. J., Morano, M., Lilienfeld, S. O., \& Poythress, N. G. (2014). Development and validation of triarchic construct scales from the Psychopathic Personality Inventory. Psychological Assessment, 26, 447-461. http:// dx.doi.org/10.1037/a0035665

Hare, R. D. (2003). The Hare Psychopathy ChecklistRevised ( $2^{\text {nd }}$ ed.). Toronto, Ontario, Canada: MultiHealth Systems.

Hoyle, R. H., Stephenson, M. T., Palmgreen, P., Lorch, E. P., \& Donohew, R. L. (2002). Reliability and validity of a brief measure of sensation seeking. Personality and Individual Differences, 32, 401-414.

John, O. P., Donahue, E. M., \& Kentle, R. L. (1991). The Big Five Inventory-Versions $4 a$ and 54. Berkeley, CA: University of California, Berkeley, Institute of Personality and Social Research.

Kutchen, T. J., Wygant, D. B., Tylicki, J. L., Dieter, A. M., Veltri C. O. C., \& Sellbom, M. (2017). Construct validity of the MMPI-2-RF triarchic psychopathy scales in correctional and collegiate samples. Journal of Personality Assessment, 99, 408-415. https:// doi.org/10.1080/00223891.2016.1238829

Kyranides, M. N., Fanti, K. A., Sikki, M., \& Patrick, C. J. (2017). Triarchic dimensions of psychopathy in young adulthood: Associations with clinical and physiological measures after accounting for adolescent psychopathic traits. Personality Disorders: Theory, Research, and Treatment, 8, 140-149. http:// dx.doi.org/10.1037/per0000193

Levenson, M. R., Kiehl, K. A., \& Fitzpatrick, C. M. (1995). Assessing psychopathic attributes in a noninstitutionalized population. Journal of Personality and Social Psychology, 68, 151-158. http:// dx.doi.org/10.1037/0022-3514.68.1.151 
Lilienfeld, S. O., \& Andrews, B. P. (1996). Development and preliminary validation of a self-report measure of psychopathic personality traits in noncriminal populations. Journal of Personality Assessment, 66, 488-524. http://dx.doi.org/10.1207/ s15327752jpa6603_3

Lilienfeld, S. O., \& Widows, M. R. (2005). Psychopathic Personality Inventory-Revised (PPI-R) professional manual. Odessa, FL: Psychological Assessment Resources.

Lynam, D. R., Gaughan, E. T., Miller, J. D., Miller, D. J., Mullins-Sweatt, S., \& Widiger, T. A. (2011). Assessing the basic traits associated with psychopathy: Development and validation of the Elemental Psychopathy Assessment. Psychological Assessment, 23, 108-124. https://doi.org/10.1037/a0021146

Lynam, D. R., Smith, G. T., Whiteside, S. P., \& Cyders, M. A. (2006). The UPPS-P: Assessing five personality pathways to impulsive behavior. West Lafayette, IN: Purdue University.

Miller, J. D., Crowe, M., Weiss, B., Lynam, D. R., \& Maples-Keller, J. L. (2017). Using online, crowdsourcing platforms for data collection in personality disorder research: The example of Amazon's Mechanical Turk. Personality Disorders: Theory, Research, and Treatment, 8, 26-34. http://dx.doi. org/10.1037/per0000191

Miller, J. D., \& Lynam, D. R. (2015). Psychopathy and personality: Advances and debates. Journal of Personality, 83, 585-592. https://doi.org/10.1111/jopy. 12145

Mills, J. F., Kroner, D. G., \& Forth, A. E. (2002). Measures of Criminal Attitudes and Associates (MCAA): Development, factor structure, reliability, and validity. Assessment, 9, 240-253. https:// doi.org/10.1177/1073191102009003003

Mills, J. F., Kroner, D. G., \& Hemmati, T. (2004). The Measures of Criminal Attitudes and Associates (MCCA): The prediction of general and violent recidivism. Criminal Justice and Behavior, 31, 717733. https://doi.org/10.1177/0093854804268755

Murphy, B. M., Skeem, J., \& Edens, J. F. (2016). Are fearless dominance traits superfluous in operationalizing psychopathy? Incremental validity and sex differences. Psychological Assessment, 28, 1597-1607. http://dx.doi.org/10.1037/pas0000288

Paolacci, G., \& Chandler, J. (2014). Inside the Turk: Understanding Mechanical Turk as a participant pool. Current Directions in Psychological Science, 23, 184188. https://doi.org/10.1177/0963721414531598

Patrick, C. J. (2010). Operationalizing the triarchic conceptualization of psychopathy: Preliminary description of brief scales for assessment of boldness, meanness, and disinhibition. Unpublished manual, Department of Psychology, Florida State University, Tallahassee, FL.

Patrick, C. J. (2018). Psychopathy as masked pathology. In C. J. Patrick (Ed.), Handbook of psychopathy (2nd ed., pp. 3-21). New York, NY: Guilford.
Patrick, C. J., \& Drislane, L. E. (2014). Triarchic model of psychopathy: Origins, operationalizations, and observed linkages with personality and general psychopathology. Journal of Personality, 83, 627643. https://doi.org/10.1111/jopy.12119

Patrick, C. J., Fowles, D. C., \& Krueger, R. F. (2009). Triarchic conceptualization of psychopathy: Developmental origins of disinhibition, boldness, and meanness. Development and Psychopathology, 21, 913-938.

Patton,J.H.,Stanford, M.S., \& Barratt, E.S.(1995). Factor structure of the Barratt Impulsiveness Scale. Journal ofClinical Psychology, 51, 768-774. http://dx.doi. org/10.1002/1097-4679(199511)51:6<768::AIDJCLP2270510607>3.0.CO;2-1

Pechorro, P., DeLisi, M., Alberto, I., Ray, J. V., \& Simoes, M. R. (2018). The triarchic model of psychopathy among incarcerated male youths: A psychometric study. Retrieved from https://www. researchgate.net/publication/328126701

Pechorro, P., Simoes, M. R., Alberto, I., \& Ray, J. V. (2018). Triarchic model of psychopathy: A brief measure among detailed female youths. Deviant Behavior, 39, 1497-1506. https://doi.org/10.1080/0 1639625.2018.1487171

Podsakoff, P. M., MacKenzie, S. B., Lee, J., \& Podsakoff, N. P. (2003). Common method biases in behavioral research: A critical review of the literature and recommended remedies. Journal of Applied Psychology, 88, 879-903. http://dx.doi. org/10.1037/0021-9010.88.5.879

Poy, R., Segarra, P., Esteller, A., Lopez, R., \& Molto, J. (2014). FFM description of the triarchic conceptualization of psychopathy in men and women. Psychological Assessment, 26, 69-76. http://dx.doi. org/10.1037/a0034642

Sellbom, M., Drislane, L. E., Johnson, A. K., Goodwin, B. E., Phillips, T. R., \& Patrick, C. J. (2016). Development and validation of MMPI-2-RF scales for indexing triarchic psychopathy constructs. Assessment, 23, 527-543. https://doi.org/10.1177/1073191115590853

Semel R. A. (2018). The Abbreviated Psychopathy Measure (APM): Two pilot studies of a brief measure of psychopathy traits. International Journal of Psychological Studies, 10. https://doi.org/10.5539/ ijps.v10n2p41

Shapiro, D. N., Chandler, J., \& Mueller, P. A. (2013). Using Mechanical Turk to study clinical populations. Clinical Psychological Science, 1, 213-220. http://dx.doi.org/10.1177/2167702612469015

Simms, L. J., Goldberg, L. R., Roberts, J. E., Watson, D., Welte, J., \& Rotterman, J. H. (2011). Computerized adaptive assessment of personality disorder: Introducing the CAT-PD project. Journal of Personality Assessment, 93, 380-389. https://doi.org/10.10 80/00223891.2011.577475

Skeem, J. L., Polaschek, D. L. L., Patrick, C. J., \& Lilienfeld, S. O. (2011). Psychopathic personality: Bridg- 
ing the gap between scientific evidence and public policy. Psychological Science in the Public Interest, 12, 95-162. http://dx.doi.org/10.1177/1529100611426706

Stanley, J. H., Wygant, D. B., \& Sellbom, M. (2013). Elaborating on the construct validity of the Triarchic Psychopathy Measure in a criminal offender sample. Journal of Personality Assessment, 95, 343350. https://doi.org/10.1080/00223891.2012.735302

Steinberg, L., Sharp, C., Stanford, M. S., \& Tharp, A. T. (2013). New trick for an old measure: The development of the Barratt Impulsiveness Scale-Brief (BIS-Brief). Psychological Assessment, 25, 216-226. http://dx.doi.org/10.1037/a0030550

Strickland, C. M., Drislane, L. M., Lucy, M., Krueger, R. F., \& Patrick, C. J. (2013). Characterizing psychopathy using DSM-5 personality traits. Assessment, 20, 327338. https://doi.org/10.1177/10733191113486691

Tapscott, J. L., Vernon, P. A., \& Veselka, L. (2012). A comparison of the construct validity of two alternative approaches to the assessment of psychopathy in the community. Journal of Personality Assessment, 94, 541-554. https://doi.org/10.1080/00223891.2012. 668595

Tellegen, A. (1995/2003). Multidimensional Personality Questionnaire-276 (MPQ-276) test booklet. Minneapolis, MN: University of Minnesota Press.

Tonnaer, F., Cima, M., Sijtsma, K., Uzieblo, K., \& Lilienfeld, S. O. (2013). Screening for psychopathy: Validation of the Psychopathic Personality Inventory-Short Form with reference scores. Journal of Psychopathology and Behavioral Assessment, 35, 153161. http://dx.doi.org/10.1007/s10862-012-9333-2

Venables, N. C., Hall, J. R., \& Patrick, C. J. (2014). Differentiating psychopathy from antisocial personality disorder: A triarchic model perspective. Psychological Medicine, 44, 1005-1013.

Weidacker, K., O'Farrell, K. R., Gray, N. S., Johnston, S. J., \& Snowden, R. J. (2017). Psychopathy and impulsivity: The relationship of the triarchic model of psychopathy to different forms of impulsivity in offenders and community participants. Personality and Individual Differences, 114, 134139. http://dx.doi.org/10.1016/j.paid.2017.03.069 


\section{APPENDIX}

Abbreviated Psychopathy Measure-2

\section{Items}

\section{Boldness}

1. I enjoy competition, and I always expect to win.

2. I prefer safety and security rather than daring and adventure. (R)

3. I have a very strong and dominating personality.

4. I always stay incredibly cool and calm no matter what the situation.

5. I can persuade anyone to go along with my ideas.

6. I am willing to take great risks in life without fear of loss or failure.

7. I could be a star in a reality TV adventure show like "Survivor".

8. Among my talents, I am excellent at being a leader and organizer.

9. I approach life with a great sense of self-confidence.

10. I sometimes have doubts about my ability to handle challenges that I face. (R)

11. I am well able to manage even the most stressful situations.

\section{Meanness}

12. Bullying, threatening, or use of force are tactics I sometimes use to obtain what I want.

13. I can be good at pretending to be nice or to care about people when it suits my purposes.

14. I would not get pleasure or excitement from hurting someone who might have insulted or offended me. (R)

15. The world is a place of competition for resources, and I intend to get and keep all I can for myself.

16. I have made deliberate efforts to harm the reputation of others at work or in my personal/social life.

17. Having a close or caring relationship with a friend, relative, or partner is important to me. (R)

18. I have intentionally caused physical pain to others.

19. It would be hard for me to be cold and callous to other people's feelings. (R)

20. I would not enjoy having people cower, cringe, or tremble before me. (R)

21. The truth is, I don't regret anything I do that others might consider wrong.

22. I usually feel some sorrow or sympathy, even if just briefly, when I hear about a tragedy that happened to a person or to people. $(\mathrm{R})$

\section{Disinhibition}

23. I tend to act quickly without thinking about the consequences, and this sometimes gets me in trouble.

24. I am an easily bored and restless type of person who needs excitement and stimulation.

25. I have impulsively stolen things.

26. I carefully think things through before I make important decisions. (R)

27. I don't mind if I break the law as long as I don't get caught.

28. I am a patient and even-tempered type of person. (R)

29. I got into considerable trouble for my behavior during my youth, and I have continued to violate social norms. 
30. I am a responsible and trustworthy type of person. (R)

31. I often become frustrated or irritated when there is any delay to getting what I want.

32. When I am upset, I am more likely to act impulsively and do things I might later regret.

33. On multiple occasions I have engaged in risky behaviors (examples: binge-drinking; driving under the influence; illicit drug use; unprotected sex; vandalism; car speeding/racing).

Note. $\mathrm{R}$ - item is reverse coded. 